Research Article

\title{
Deep Learning-Based CT Imaging in Perioperative Period and Nursing of Esophageal Carcinoma Patients
}

\author{
Qiaoli Wang (iD) ${ }^{1}$ and Jinfu Zhu $\mathbb{i}^{2}$ \\ ${ }^{1}$ Department of Anesthesiology and Perioperative Medicine, The First Affiliated Hospital of Nanjing Medical University, \\ Nanjing 210029, China \\ ${ }^{2}$ Department of Cardiovascular Surgery, The First Affiliated Hospital of Nanjing Medical University, Nanjing 210029, China \\ Correspondence should be addressed to Jinfu Zhu; zhujingfu@njmu.edu.cn
}

Received 12 May 2021; Revised 13 June 2021; Accepted 30 June 2021; Published 29 July 2021

Academic Editor: Gustavo Ramirez

Copyright (c) 2021 Qiaoli Wang and Jinfu Zhu. This is an open access article distributed under the Creative Commons Attribution License, which permits unrestricted use, distribution, and reproduction in any medium, provided the original work is properly cited.

\begin{abstract}
The study focused on the performance of Convolutional Neural Network- (CNN-) based lymph node recognition model as well as the effects of different rehabilitation nursing methods on patients with esophageal cancer. Specifically, the activation function and loss function were optimized by CNN, to establish a U-Net lymph node recognition model. It was compared with Mean Shift and Fuzzy C-means (FCM) algorithm for the loss value, the mean pixel accuracy (mPA), and intersection over union (IOU). 158 patients with esophageal cancer undergoing radical resection were selected as research subjects. With pathological diagnosis results as the gold standard, the role of CT imaging was evaluated in the diagnosis of esophageal cancer lymph nodes. All subjects were divided into control group (routine nursing) and intervention group (routine nursing + rehabilitation nursing) according to different nursing methods, with 79 cases in each. The two groups were compared in terms of the time in bed, hospital stay, indwelling chest tube time, and VAS scores. It was found that the loss value of the U-Net model was close to 0 when it was stable, and its IOU value and mPA value were significantly higher than those of the Mean Shift and FCM algorithms. The accuracy, sensitivity, specificity, positive predictive value, and negative predictive value of the U-Net model were $84.37 \%, 80.74 \%, 88.65 \%$, $85.02 \%$, and $87.16 \%$, respectively. When it came to lymph node metastasis number of $1-2$, there were notable differences between $\mathrm{CT}$ results and postoperative pathology results, and the difference was statistically significant $(P<0.05)$. As for their identifying lymph node metastasis area, there was no statistically significant difference $(P>0.05)$. The intervention group exhibited lower postoperative VAS score, shorter time in bed, and shorter hospital stay and indwelling chest tube time versus the control group $(P<0.01)$. It suggested that the U-Net model optimized by CNN has high diagnostic efficiency for lymph nodes, and the rehabilitation nursing intervention significantly mitigates postoperative pain and accelerates postoperative recovery.
\end{abstract}

\section{Introduction}

Esophageal carcinoma is one of the common malignant tumors originating in the digestive tract. It mainly develops in the glands or mucosal epithelium of the esophagus and is one of the top ten malignant tumors with high incidence. The esophageal carcinoma is highly prevalent in China, and both of its morbidity and mortality rates rank first worldwide. An estimated 200,000 population are diagnosed with esophageal carcinoma each year, and approximately 15.32-20.78/100,000 people die of esophageal carcinoma each year [1]. The current treatment of esophageal cancer is surgical resection, but long-term effects of surgical resection are not good, with only about $30 \%$ survival rate within 5 years [2]. An abundance of research results reveals that lymph node metastasis is one of the most important factors affecting the prognosis of esophageal carcinoma patients, and the number of lymph node metastases is a key factor for the prognosis of esophageal carcinoma patients [3]. Therefore, to accurately identify the lymph node metastasis number and the metastatic area is of great significance to the surgical treatment and prognosis of esophageal carcinoma.

In recent years, a number of diagnostic methods such as CT, Magnetic Resonance Imaging (MRI), endoesophageal 
ultrasound, and esophageal endoscopy have been used in diagnosis of esophageal carcinoma as a result of the rapid progression of medical imaging technology. Among them, $\mathrm{CT}$ is characteristic of non-invasiveness and high accuracy, and it is superior to other methods in the diagnosis of esophageal carcinoma, the identification of foci, and the judgment of lymph node metastasis [4]. Clinical data have found that conventional CT scanning images rely on professional knowledge of physicians to extract and label lymph nodes, which is subjective to a certain extent, and the detection rate is quite different from that of the pathological diagnosis of lymph nodes at different locations [5]. Thanks to the development of deep learning, many researchers have applied various deep learning algorithms in the diagnosis of CT images. At present, the organ segmentation algorithms mainly include region growing method, clustering method, Mean Shift segmentation method, and CNN segmentation method [6]. Among them, CNN demonstrates superb capabilities in retaining spatial information of the image, which lays a solid foundation for the segmentation of organs in the focal area. Although $\mathrm{CNN}$ has found broad applications in the segmentation of medical images, most research focuses on the MRI-based human brain image segmentation. There is little research on CT image segmentation, let alone esophageal CT image segmentation.

In summary, the esophageal carcinoma lymph node metastasis number and the metastasis area have important implications for surgical methods and prognosis. CT, as one of the important methods for the diagnosis of esophageal carcinoma, still has certain limitations in the diagnosis of lymph node metastasis. In the study, CNN was used to optimize the lymph node recognition model, which was applied to the diagnosis and prognosis analysis of esophageal cancer. Patients with esophageal cancer were selected as the research subjects, and they were grouped based on different nursing methods. The primary objective of the study was to investigate the role of CNN-based CT imaging technology in diagnosis of esophageal cancer, and the secondary aim was to explore the effects of different nursing methods.

\section{Materials and Methods}

2.1. Research Subjects and Grouping. 158 patients with esophageal carcinoma who underwent radical resection in hospital from September 2017 to April 2020 were selected as the research subjects. Among them, there were 102 males and 56 females, aged between 23 and 79 years old, and the average age was $(60.84 \pm 8.33)$. They were randomly divided into the control group and intervention group according to different nursing methods, with 79 in each. The inclusion criteria of this study were as follows: (i) patients with esophageal cancer undergoing radical resection; (ii) patients with no history of malignant tumors; (iii) patients having chest and abdomen CT examinations before and after surgery; and (iv) patients not receiving radiotherapy and chemotherapy before or after surgery. The exclusion criteria were as follows: (i) those with severe disease involving important organs; (ii) those who have received radiotherapy or chemotherapy before surgery; (iii) those who refused surgical treatment; and (iv) those who had incomplete patient examination and surgical data. The experimental procedure has been approved by the ethics committee of hospital, and all subjects included in the study had signed an informed consent form.

2.2. The Image Segmentation Algorithm Based on CNN. $\mathrm{CNN}$ is an end-to-end fully convolutional image segmentation neural network based on deep learning [7]. For $n$ sample points in the $d$-dimensional space $x_{i}, i=1,2, \ldots, n$, the calculation method for the dimensional numerical vector $M_{k}$ at any point $x$ is as follows:

$$
M_{k}=\frac{1}{k} \sum_{x_{i} \in S_{k}}\left(x_{i}-x\right),
$$

where $S_{k}$ represents a $d$-dimensional spherical area with a radius of $r, k$ is the number of sample points that fall into the spherical area, and $\left(x_{i}-x\right)$ represents the relative offset between the sample points $x_{i}$ and the reference point $x$. The set of all sample points in this spherical area is then expressed as follows:

$$
S_{k}(x)=\left\{y:\left(y-x_{i}\right)^{T}\left(y-x_{i}\right)<r^{2}\right\} .
$$

For a two-dimensional matrix $A$, the matrix coordinate point $(x, y)$ is subjected to convolution operation, as shown in equation (3), where $K$ is the convolution kernel; $S$ is the convolution result; $m$ is the number of neurons in the input layer; and $n$ is the number of neurons in the output layer.

$$
S(x, y)=(K * A)(x, y)=\sum_{m} \sum_{n} A(x-m, j-n) K(m, n) .
$$

After compressing the CT image and extracting its features, the effective features of the compressed image can be expressed as follows:

$$
\begin{aligned}
& W_{2}=\frac{\left(W_{1}-W_{F}+2 P\right)}{B+1}, \\
& H_{2}=\frac{\left(H_{1}-H_{F}+2 P\right)}{B+1},
\end{aligned}
$$

where $W_{2}$ is the width of the output feature map; $H_{2}$ is the height of the output feature map; $W_{1}$ and $H_{1}$ are the width and height of the input feature map and $W_{F}$ and $H_{F}$ are the width and height of the convolution kernel, respectively; $P$ is the number of pixel values filled on the input feature image; and $B$ is the step size of the convolution kernel.

Assuming that the input item of a certain segment of neural network is $x$, the expected complex latent mapping is represented by $H(x)$, and the shortcut connection is used to directly pass the input $x$ to the output, which can be expressed as follows:

$$
H(x)=f(x)+x .
$$

The activation function endows the neural network with nonlinear learning ability and improves the model's ability 
to express nonlinear relationships [8]. In the study, ReLU is used as the basic activation function. The expression of ReLU nonlinear activation function is as follows:

$$
f(x)=\max (0,1) .
$$

Compared with the traditional Sigmoid and tanh activation functions, ReLU can accelerate the training of the model [9]. After optimization, it is expressed as follows:

$$
f\left(x_{i}\right)= \begin{cases}x_{i}, & x_{i}>0, \\ a_{i} x_{i}, & x_{i} \leq 0,\end{cases}
$$

where $a_{i}$ is a learnable parameter and $i$ is the channel number. $a_{i}$ can learn using the backpropagation algorithm, and the propagation learning method can be expressed as follows:

$$
\frac{\partial \eta}{\partial a_{i}}=\sum_{x_{i}} \frac{\partial \eta}{\partial f\left(x_{i}\right)} \frac{\partial f\left(x_{i}\right)}{\partial a_{i}}
$$

where $\eta$ is the learning rate, and $\partial f\left(x_{i}\right) / \partial a_{i}$ needs to meet the conditions $\left(\partial f\left(x_{i}\right) / \partial a_{i}\right)=\left\{\begin{array}{ll}0 & x_{i}>0 \\ x_{i} & x_{i} \leq 0\end{array}\right.$.

In the training phase, the Softmax loss function is used to update the weight of the loss of the sample. Softmax can update and optimize the weight parameters without interruption, and the normalization operation in the Softmax classifier makes the value more stable [10]. The optimized $\mathrm{CNN}$ algorithm is named U-Net algorithm. The Softmax loss function can be expressed as follows:

$$
\left[\begin{array}{c}
p\left(y^{i}=1 \mid x^{i} ; \theta\right) \\
\vdots \\
p\left(y^{i}=k \mid x^{i} ; \theta\right)
\end{array}\right]=\frac{1}{\sum_{j=1}^{k} e^{\theta_{j}^{T} x^{i}}}\left[\begin{array}{c}
e^{\theta_{1}{ }^{T} x^{i}} \\
\vdots \\
e^{\theta_{k}{ }^{T} x^{i}}
\end{array}\right],
$$

where $k=4,\left(x^{i}, y^{i}\right)$ is the data training set, $y^{i} \in[1,2 \ldots, k]$, and $p$ represents the probability of each category.

In the study, the commonly used image segmentation evaluation index mPA and IOU are used to quantitatively analyze the organ segmentation effects [11]. They are calculated as follows:

$$
\begin{aligned}
& \mathrm{mPA}=1-\frac{A(\text { xor }) B}{m * n}, \\
& \mathrm{IOU}=\frac{Y_{i} \cap X_{i}}{Y_{i} \cup X_{i}},
\end{aligned}
$$

where $A$ and $B$ are the label image and segmentation results, respectively; xor represents the exclusive OR operation between image $A$ and image $B ; m$ and $n$ represent the length and width of the image, respectively; and $Y_{i}$ and $X_{i}$ represent the area occupied by an organ and the corresponding area occupied by the segmented organ.

2.3. Lymph Node Recognition Process of Esophageal Carcinoma Based on CNN Algorithm. In this study, an esophageal cancer lymph node recognition model was established based on the $\mathrm{CNN}$ image segmentation algorithm. The specific process is shown in Figure 1. The initial CT image was preprocessed by removing the examination bed and subcutaneous tissue, and then the U-Net CT was used to extract the esophagus region, followed by further extraction of the region of interest. Finally, the topological relationship between the suspected lymph node and the surrounding tissue was identified, and the suspected lymph node was extracted.

2.4. CT Scan Conditions and Diagnostic Criteria. Before the CT scanning, the patient was instructed to lie supine. The scan range mainly included the lower neck, supraclavicular, chest, and upper abdomen. During the scan, the patient needed to inhale deeply and hold the breath. CT scan parameters were as follows: $120 \mathrm{kv}, 90 \mathrm{~mA}$, collimation of $2.5 \sim 5.0 \mathrm{~cm}$, screw pitch of $25 \mathrm{~mm}$, bed speed of $50 \mathrm{~mm} / \mathrm{s}$, layer thickness of $3.0 \sim 6.0 \mathrm{~mm}$, and layer spacing of $3.0 \sim 6.0 \mathrm{~mm}$. Non-ionic iohexol injection was used as a contrast agent for enhanced scanning. The injection dose was calculated at $1.5 \mathrm{~mL} 2 \mathrm{~mL} / \mathrm{kg}$, the injection rate was $2.0 \sim 3.0 \mathrm{~mL} / \mathrm{s}$, and the scan started 30 seconds after injection. The CT images of all patients were transferred to the threedimensional treatment planning system to obtain the required indexes.

The diagnostic criteria for esophageal lesions are as follows: the thickness of the esophageal wall more than $5 \mathrm{~mm}$ and the diameter of the airless esophagus greater than $10 \mathrm{~mm}$; the diagnostic criteria for lymph node metastasis referred to the diagnostic criteria in the study of Kashima et al. [12].

2.5. Perioperative Nursing Methods and Observation Indexes for Esophageal Carcinoma. The patients in the control group were treated with routine care after esophageal carcinoma surgery. The intervention group patients accepted rapid rehabilitation referring to research of Borrett [13] on the basis of routine nursing, during which a series of measures such as intraoperative heat preservation and postoperative analgesia were adopted.

A number of indexes were observed for the postoperative time to get out of bed, hospital stay, and indwelling chest tube duration to evaluate the pain degree at different time periods after the operation. The pain evaluation referred to the method of Wang et al. [14] and used the Visual Analogue Scale (VAS) to evaluate.

2.6. Statistics. Experimental data were processed using SPSS19.0. Measurement data were calculated as mean\pm standard deviation $( \pm s)$, and count data were expressed in percentage (\%). The $\chi^{2}$ test was applied. $P<0.05$ was set as the threshold for significance.

\section{Results}

3.1. Analysis of Organ Segmentation Ability. The improved and optimized U-Net network was compared with Mean Shift and Fuzzy C-means (FCM) organ segmentation algorithms for the loss function (loss) curve. As shown in 


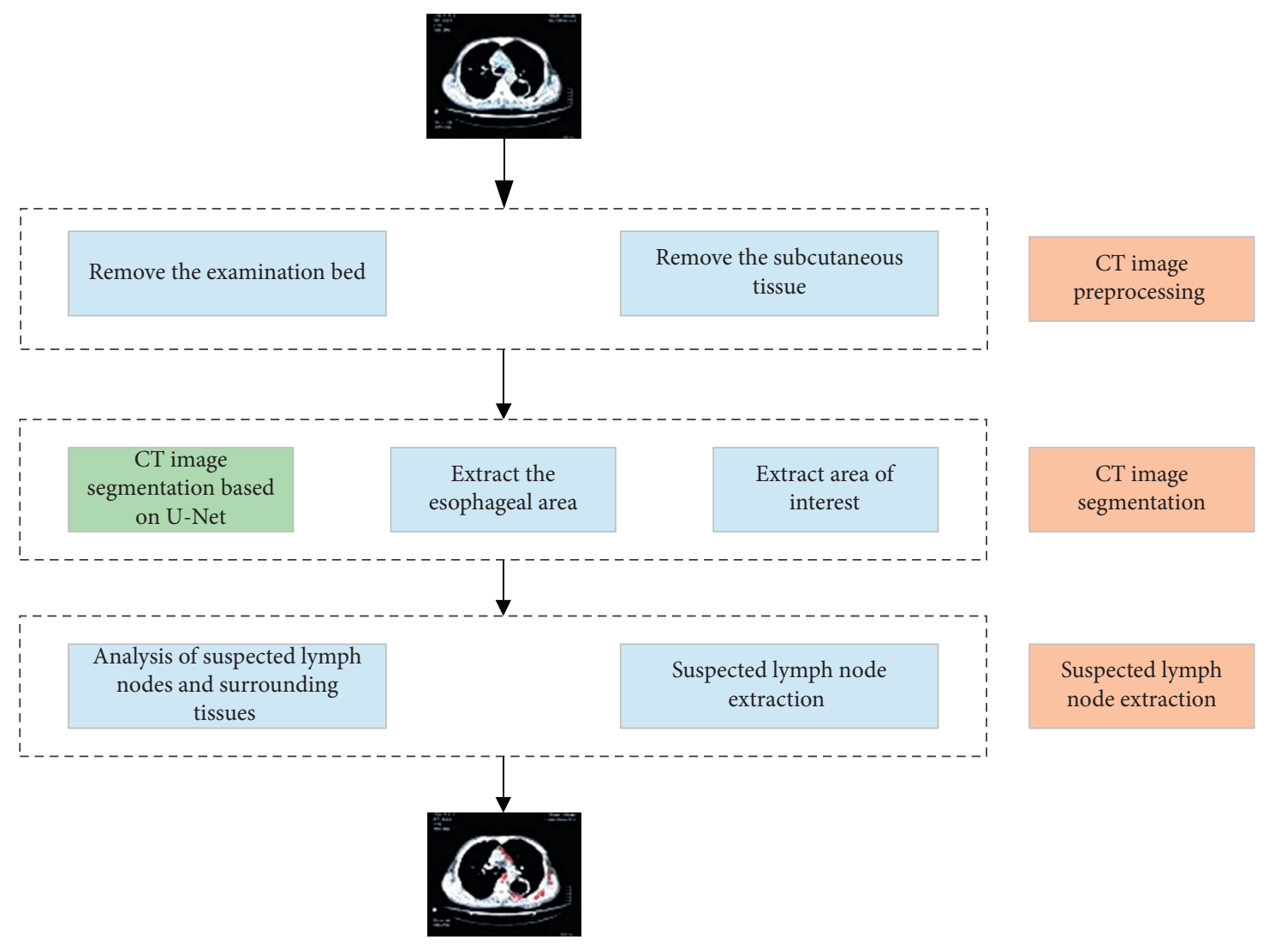

FIGURE 1: Lymph node recognition process based on CNN algorithm.

Figure 2, the loss function of U-Net was superior to that of the Mean Shift and FCM methods in terms of convergence speed, and the loss of the FCM method was stable around 0.1 . When the epoch was 50, the loss of the Mean Shift method was stable around 0.05 , while the loss of the optimized U-Net was stable around 0 under the same conditions.

The IOU curves were compared and analyzed between different algorithms. As shown in Figure 3, the IOU value of the U-Net algorithm was notably higher versus the Mean Shift and FCM methods.

The comparison of the mPA of CT images of different algorithms showed that the U-Net algorithm exhibited higher mPA value versus the Mean Shift, FCM, and Fully Convolutional Network (FCN) methods, as shown in Figure 4.

\subsection{Analysis of Suspected Lymph Node Detection Results.} The suspected lymph node detection method based on the U-Net algorithm was applied. As shown in Figure 5, the CT diagnosis revealed that 95 cases $(60.13 \%)$ with esophageal cancer did not show lymph node metastasis (the metastasis number was 0), 54 cases (34.17\%) had 1-2 lymph node metastases, and 9 cases (5.70\%) showed lymph node metastases greater than 3 . The pathological diagnosis results illustrated that there are 108 cases $(68.35 \%)$ with esophageal cancer exhibiting no lymph node metastasis, 36 patients $(22.78 \%)$ had lymph node metastases of $1-2$, and $14(8.86 \%)$ patients had lymph node metastases greater than 3 . There

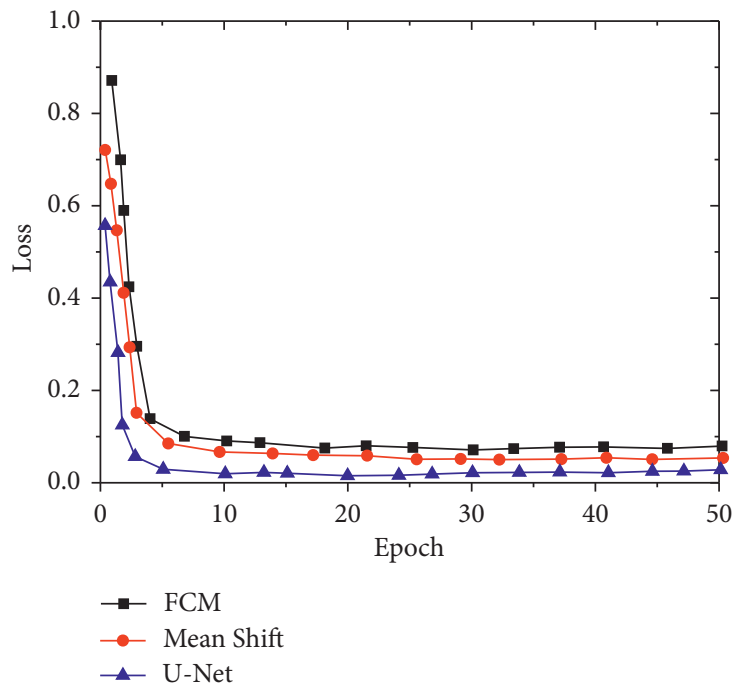

Figure 2: Comparison of loss curves.

were notable differences in diagnosis for 1-2 lymph node metastases between CT and postoperative pathology diagnosis $(P<0.05)$.

CT images were used to diagnose suspicious lymph nodes in patients with esophageal cancer. As shown in Figure 6, CT images can significantly identify the tracheal carina and the right trachea paraesophageal groove lymph nodes. 


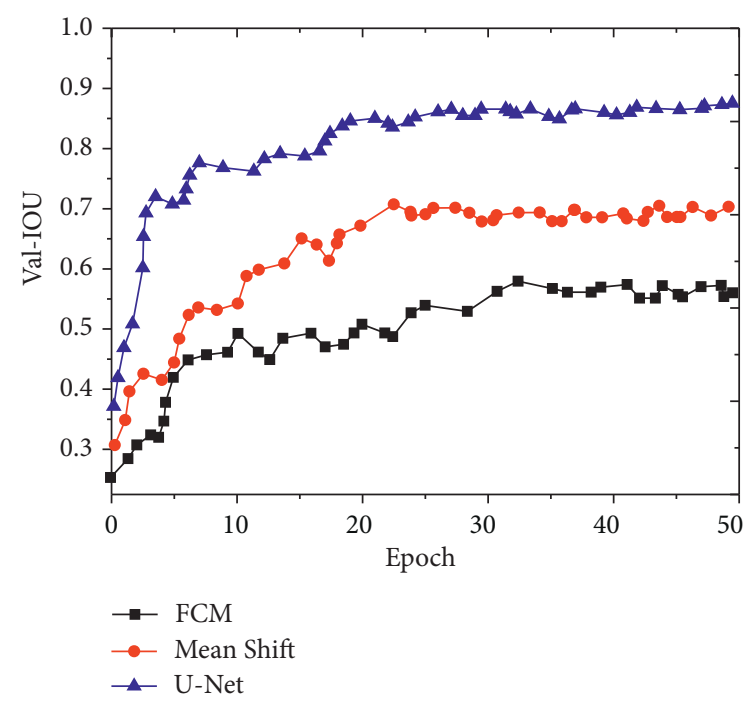

FIgURE 3: Comparison of IOU curves.

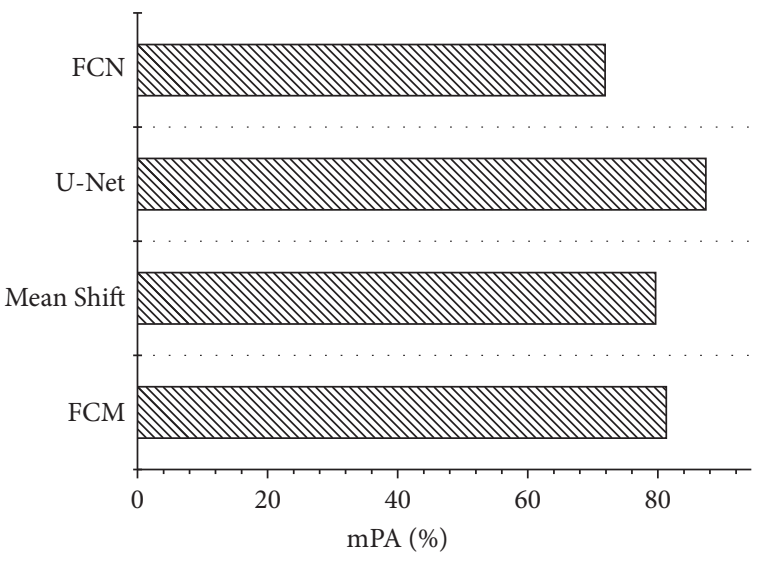

Figure 4: Comparison of mPA values.

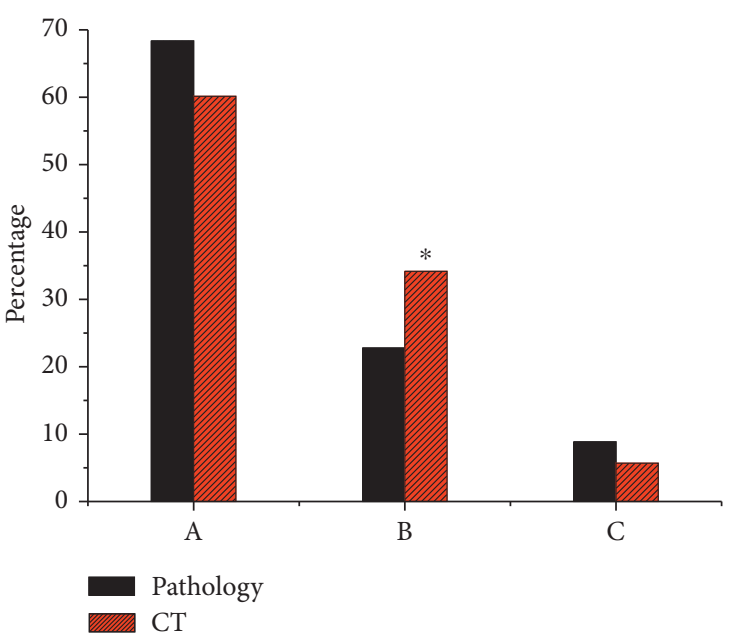

FIGURE 5: Comparison of CT diagnosis and postoperative pathological diagnosis for suspicious lymph nodes (A: 0 lymph node metastasis; B: 1-2 lymph node metastases; C: more than 3 lymph node metastases; ${ }^{*}$ a notable difference in comparison to the pathological diagnosis result, $P<0.05$ ).
3.3. Analysis of the Efficacy of CT Imaging in the Diagnosis of Suspected Lymph Nodes in Esophageal Cancer. The suspected lymph node metastasis area identified by CT imaging and the pathological diagnosis was as shown in Figure 7. The pathological diagnosis results revealed that there were 33 patients $(20.89 \%)$ with lymph node metastasis in 1 area, and 17 patients $(10.76 \%)$ exhibited lymph node metastasis with more than 2 areas involved. CT imaging showed that 40 patients $(25.32 \%)$ had lymph node metastasis in one area, and 23 patients $(14.56 \%)$ had lymph node metastasis in more than two areas. The difference between CT and postoperative pathology in the diagnosis of lymph node metastasis area was not notable $(P>0.05)$.

Based on the gold standard of pathological diagnosis, the effectiveness of CT imaging in diagnosing suspicious lymph nodes in esophageal cancer was analyzed. As shown in Figure 8, the accuracy, sensitivity, specificity, positive predictive value, and negative predictive value of CT imaging in diagnosing suspicious lymph nodes in esophageal cancer were $84.37 \%, 80.74 \%, 88.65 \%, 85.02 \%$, and $87.16 \%$, respectively.

3.4. Comparison of Pain Degree after Different Nursing Methods. After different nursing methods were adopted, the pain degree at different time periods was compared. As shown in Figure 9, both groups of patients had the highest VAS scores at 6th hour after surgery. As time went by, the VAS scores of the two groups showed a downward trend, and during the entire observation period, the VAS scores of the intervention group were lower versus the control group $(P<0.01)$.

3.5. Comparison of Postoperative Recovery Time between the Two Groups. The time needed to get out of bed after the surgery was analyzed. As shown in Figure 10, in the intervention group, the time to get out of bed was shorter $(28.76 \pm 1.96$ versus $52.92 \pm 4.13)(P<0.01)$.

The postoperative chest tube duration and bowel movement recovery time of the two groups were analyzed. As shown in Figure 11, the postoperative chest tube duration and bowel movement recovery time of the intervention group were shorter $(P<0.01)$.

The postoperative hospital stay of the two groups was analyzed. As shown in Figure 12, the postoperative hospital stay of the intervention group was shorter $(8.03 \pm 0.63$ versus $9.45 \pm 0.74)(P<0.01)$.

\section{Discussion}

Mean pixel accuracy (mPA) is an index defined as the ratio of correctly classified pixels to the total number of pixels and is one of the commonly used evaluation indexes for image segmentation [15]. The IOU is an important index to measure the accuracy of image segmentation [16]. In the study, the improved and optimized U-Net was compared with Mean Shift and FCM segmentation algorithms. It was found that the IOU value of U-Net algorithm was notably 

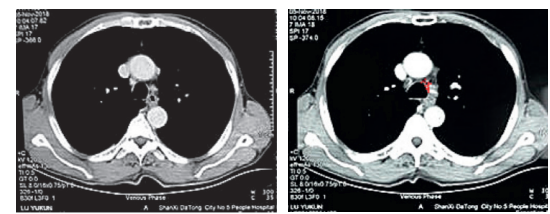

(a)
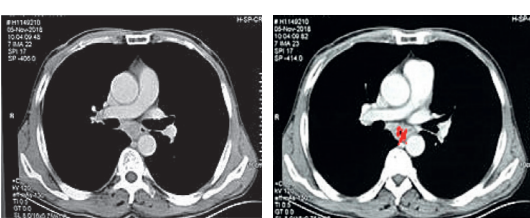

(b)

FIGURE 6: Identification of suspicious lymph nodes in esophageal carcinoma by CT images. (a) 65-year-old male's tracheal carina lymph nodes: (A) original CT scan; (B) suspected lymph node labeling. (b) 36-year-old male's right tracheal paraesophageal groove lymph node: (C) original CT scan; (D) suspected lymph node labeling.

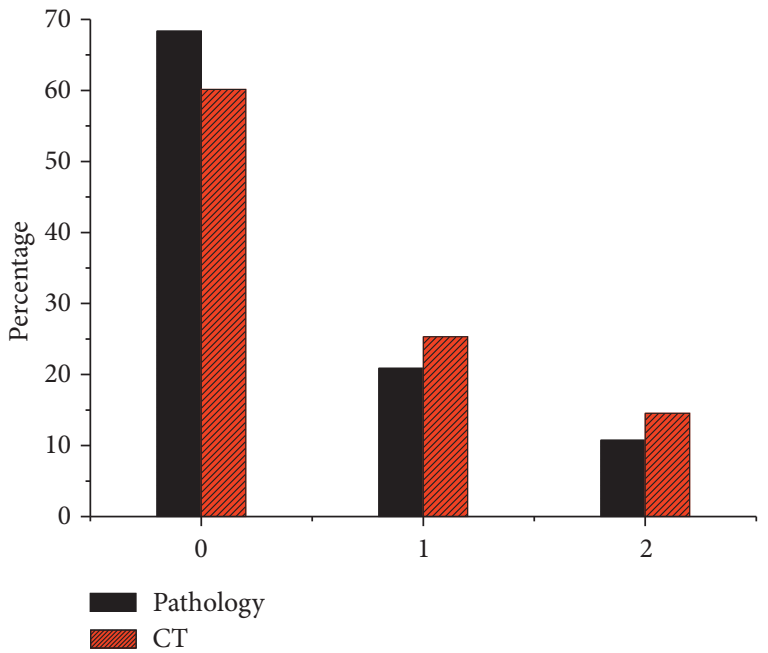

Figure 7: Comparison of suspicious lymph node area identified by $\mathrm{CT}$ and postoperative pathological diagnosis.

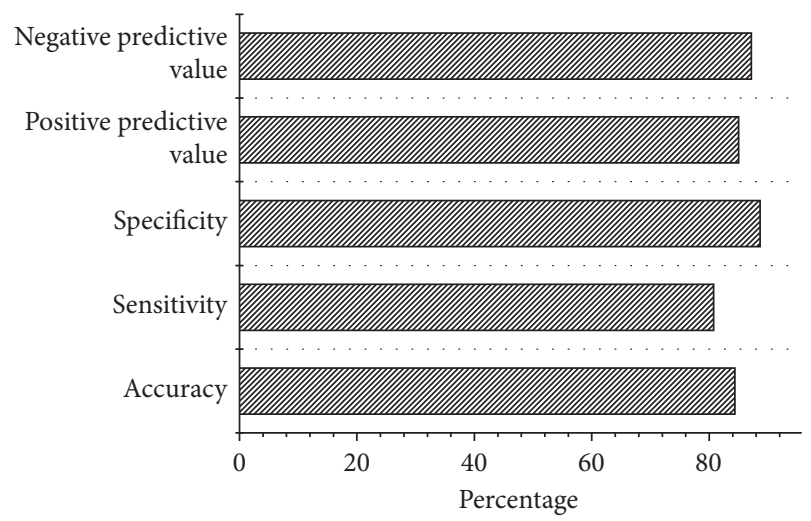

FIgUre 8: Analysis of the effectiveness of CT in diagnosing suspicious lymph nodes.

higher than that of Mean Shift and FCM, and it showed a higher mPA value versus Mean Shift, FCM, and FCN. Taken together, these results suggested that the optimized U-Net algorithm demonstrated better organ segmentation effects.

The lymphatic network of the esophageal lymphatic system is relatively rich. The diagnosis results of ordinary CT for the esophageal carcinoma lymph node metastasis are quite different from the pathological results [17]. In this study, the diagnosis results of the lymph node metastasis

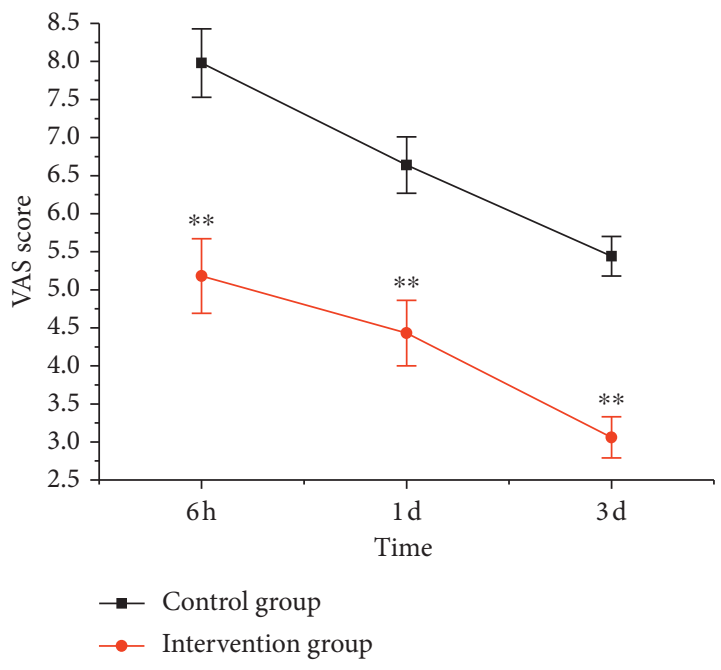

Figure 9: Comparison of postoperative pain $\left({ }^{* *}\right.$ a notable difference in comparison to the control group, $P<0.01$ ).

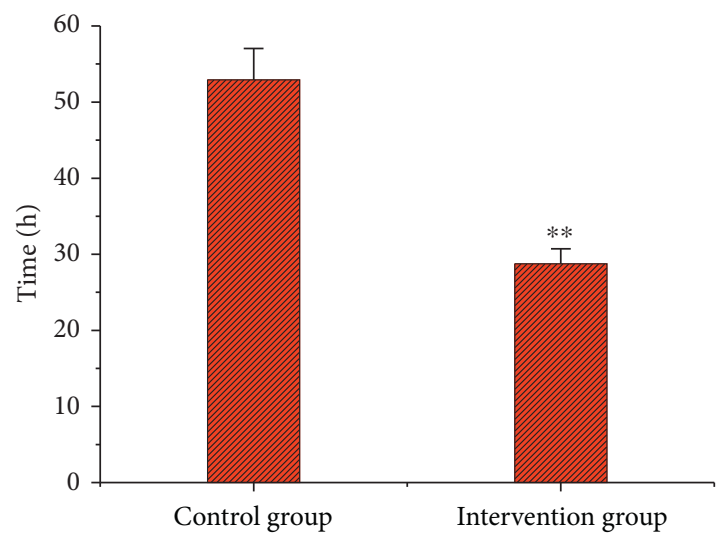

FIgURE 10: Comparison of time needed to get out of bed $\left({ }^{* *}\right.$ indicates a notable difference in comparison to the control group, $P<0.01$ ).

based on the U-Net segmentation algorithm found that there were notable differences in diagnosis for 1-2 lymph node metastases between $\mathrm{CT}$ diagnosis and postoperative pathological diagnosis $(P<0.05)$. The CT diagnosis and postoperative pathology diagnosis showed no notable differences in the diagnosis of lymph node metastasis area $(P>0.05)$. It was evident that CT imaging exhibited a higher number of false-negative lymph nodes in the diagnosis of suspicious 


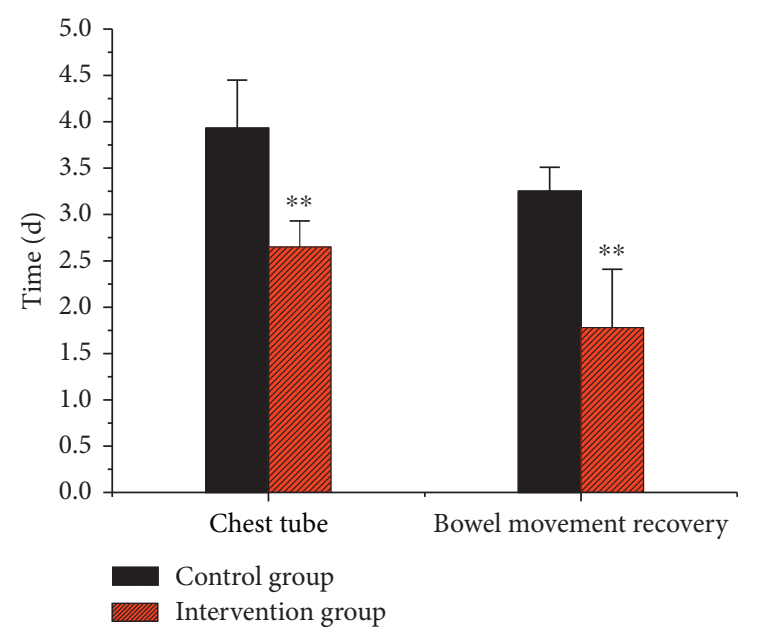

FIgURE 11: Comparison of postoperative chest tube duration and bowel movement recovery time $\left({ }^{* *}\right.$ indicates a notable difference in comparison to the control group, $P<0.01$ ).

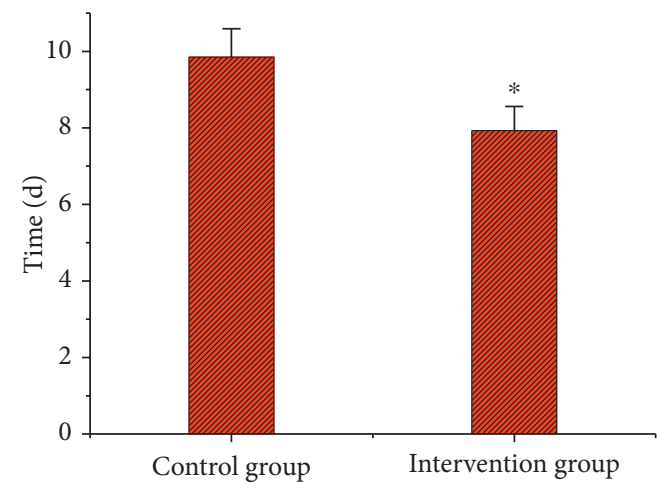

FIGURE 12: Comparison of postoperative hospital stay $\left({ }^{*}\right.$ indicates a notable difference in comparison to the control group, $P<0.01$ ).

esophageal carcinoma lymph nodes, which was consistent with the results of $\mathrm{Wu}$ et al. [18]. It may be because of the different standards for CT diagnosis of lymph nodes, which contributes to the deviations from the pathological results. The accuracy, sensitivity, specificity, positive predictive value, and negative predictive value of CT imaging in the diagnosis of suspicious esophageal carcinoma lymph nodes were $84.37 \%, 80.74 \%, 88.65 \%, 85.02 \%$, and $87.16 \%$, respectively, while these indexes of ordinary CT in diagnosing suspicious esophageal carcinoma lymph nodes were $71.81 \%$, $54.87 \%, 84.93 \%, 73.81 \%$, and $70.86 \%$, respectively [19]. Notably, the optimized algorithm in this study has improved the diagnostic efficiency of suspicious lymph nodes in esophageal carcinoma.

Based on the CT diagnosis results, different nursing methods were adopted to care for patients with esophageal carcinoma. It was found that the intervention group had lower postoperative VAS score, time of getting out of bed, indwelling chest tube duration, bowel movement recovery time, and postoperative hospital stay versus the control group $(P<0.01)$. It illustrated that rapid rehabilitation nursing intervention can significantly alleviate postoperative pain and speed up postoperative recovery, which was in line with the results of Wang et al. [20].

\section{Conclusion}

Aiming to explore the role of CT imaging based on deep learning in the perioperative period and nursing of esophageal carcinoma patients, an optimized U-Net segmentation algorithm was used to establish a lymph node recognition model, which was applied during the perioperative period of esophageal carcinoma patients. Based on the CT diagnosis results, different nursing methods were given and their effects on the rehabilitation were investigated. It was found that the U-Net algorithm demonstrated better organ segmentation effects, and the diagnostic efficiency was notably heightened. Rapid rehabilitation nursing was found to greatly alleviate the postoperative pain and speed up postoperative recovery. However, some shortcomings should be noted in this study. It only evaluates the diagnostic efficiency of CT images for lymph nodes and does not analyze the prognostic value. In future work, the prognostic value of CT imaging will be further analyzed, which is expected to provide reference for the prognosis of esophageal carcinoma surgery.

\section{Data Availability}

The data used to support the findings of this study are available from the corresponding author upon request.

\section{Conflicts of Interest}

The authors declare that they have no conflicts of interest.

\section{References}

[1] M. C. J. Anderegg, J. P. Ruurda, S. S. Gisbertz et al., "Feasibility of extended chemoradiotherapy plus surgery for patients with cT4b esophageal carcinoma," European Journal of Surgical Oncology, vol. 46, no. 4, pp. 626-631, 2020.

[2] F.-L. Huang and S.-J. Yu, "Esophageal cancer: risk factors, genetic association, and treatment," Asian Journal of Surgery, vol. 41, no. 3, pp. 210-215, 2018.

[3] R.-H. Wang, "From reflux esophagitis to Barrett's esophagus and esophageal adenocarcinoma," World Journal of Gastroenterology, vol. 21, no. 17, pp. 5210-5219, 2015.

[4] W.-L. Chang, W.-L. Wang, T.-J. Chung et al., "Response evaluation with endoscopic ultrasound and computed tomography in esophageal squamous cell carcinoma treated by definitive chemoradiotherapy," Journal of Gastroenterology and Hepatology, vol. 30, no. 3, pp. 463-469, 2015.

[5] K. Wakatsuki, S. Matsumoto, K. Migita et al., "Usefulness of computed tomography density of a tumor in predicting the response of advanced esophageal cancer to preoperative chemotherapy," Surgery, vol. 162, no. 4, pp. 823-835, 2017.

[6] B. Oliveira, S. Queirós, P. Morais et al., "A novel multi-atlas strategy with dense deformation field reconstruction for abdominal and thoracic multi-organ segmentation from computed tomography," Medical Image Analysis, vol. 45, pp. 108-120, 2018.

[7] M. Colevray, V. Tatard-Leitman, S. Gouttard, P. Douek, and L. Boussel, "Convolutional neural network evaluation of 
over-scanning in lung computed tomography," Diagnostic and Interventional Imaging, vol. 100, no. 3, pp. 177-183, 2019.

[8] Y. Zhang and $\mathrm{H}$. Yu, "Convolutional neural network based metal artifact reduction in x-ray computed tomography," IEEE Transactions on Medical Imaging, vol. 37, no. 6, pp. 1370-1381, 2018.

[9] D. Wu, K. Kim, and Q. Li, "Computationally efficient deep neural network for computed tomography image reconstruction," Medical Physics, vol. 46, no. 11, pp. 4763-4776, 2019.

[10] Y. Gao, Z.-D. Zhang, S. Li et al., "Deep neural networkassisted computed tomography diagnosis of metastatic lymph nodes from gastric cancer," Chinese Medical Journal, vol. 132, no. 23, pp. 2804-2811, 2019.

[11] M. L. Welch, C. McIntosh, A. Traverso et al., "External validation and transfer learning of convolutional neural networks for computed tomography dental artifact classification," Physics in Medicine and Biology, vol. 65, no. 3, Article ID 035017, 2020.

[12] H. Kashima, K. Noma, T. Ohara et al., "Cancer-associated fibroblasts (CAFs) promote the lymph node metastasis of esophageal squamous cell carcinoma," International Journal of Cancer, vol. 144, no. 4, pp. 828-840, 2019.

[13] K. Borrett, "Interventions for dysphagia in esophageal cancer," Gastroenterology Nursing, vol. 41, no. 1, pp. 73-75, 2018.

[14] Y. Wang, J. Cheng, L. Yang, J. Wang, H. Liu, and Z. Lv, "Ropivacaine for intercostal nerve block improves early postoperative cognitive dysfunction in patients following thoracotomy for esophageal cancer," Medical Science Monitor, vol. 25, pp. 460-465, 2019.

[15] S. Wang, K. He, D. Nie, S. Zhou, Y. Gao, and D. Shen, "CT male pelvic organ segmentation using fully convolutional networks with boundary sensitive representation," Medical Image Analysis, vol. 54, pp. 168-178, 2019.

[16] A. Balagopal, S. Kazemifar, D. Nguyen et al., "Fully automated organ segmentation in male pelvic CT images," Physics in Medicine and Biology, vol. 63, no. 24, Article ID 245015, 2018.

[17] Y. Zhao and Y. Mao, "Pattern of lymph node metastasis and choice of lymphadenectomy in patients with thoracic esophageal squamous cancer," Zhonghua Wei Chang Wai Ke Za Zhi, vol. 21, no. 9, pp. 987-994, 2018.

[18] H. R. Wu, C. Q. Liu, M. F. Guo, M. Q. Xu, and X. Y. Mei, "Analysis on CT in diagnosis of lymph node metastasis of thoracic esophageal cancer with minimum diameter greater than $1 \mathrm{~cm}$," Zhonghua Wai Ke Za Zhi, vol. 57, no. 8, pp. 601-606, 2019.

[19] L. Goense, J. Meziani, P. S. N. Van Rossum et al., "Limited additional value of cervical ultrasonography over a negative 18F-FDG PET/CT for diagnosing cervical lymph node metastases in patients with esophageal cancer," Nuclear Medicine Communications, vol. 39, no. 7, pp. 645-651, 2018.

[20] G. M. Wang, D. F. Liu, Y. P. Xu, T. Meng, and F. Zhu, "PET/ CT imaging in diagnosing lymph node metastasis of esophageal carcinoma and its comparison with pathological findings," European Review for Medical and Pharmacological Sciences, vol. 20, no. 8, pp. 1495-1500, 2016. 